\title{
La DS-DIBA: Experiencia de la elaboración de una herramienta de valoración social y segmentación para los servicios sociales
}

\author{
Miguel Ángel Manzano Rodríguez¹; Étienne Pagés Gaulier²; Marta Solé Pascual ${ }^{3}$
}

Recibido: 20/11/2017 / Revisado: 06/12/2017 / Aceptado: 15/06/2018

Disponible on line:

Resumen. La mejora de la eficacia y eficiencia del proceso de atención mediante la segmentación es uno de los retos principales de los servicios sociales básicos de atención primaria. Para avanzar en este objetivo se ha desarrollado un proyecto de investigación-acción de rediseño y mejora continua, en 16 municipios de la provincia de Barcelona.

El procedimento de segmentación se desarrolla mediante un instrumento de valoración social, denominado DS-DIBA, que proporciona confianza, seguridad y robustez en los diagnósticos sociales.

El principal resultado es la identificación por profesionales expertos de las situaciones personales y familiares que causan las necesidades objeto de intervención de los servicios sociales; así como de los factores de contexto que inciden. Esta herramienta describe cada situación a partir de evidencias objetivables, las clasifica según su gravedad y frecuencia. Finalmente segmenta cada caso en tres niveles de riesgo en función de la incidencia que presenta para la unidad de convivencia.

La implementación del DS_DIBA aporta valor como soporte durante el proceso de intervención social: identificando perfiles comunes entre la población usuaria; avanzando en un lenguaje compartido y estructurado y en la integración horizontal y vertical; ayudando a la focalización del caso; y priorizando la intervención en términos de intensidad de atención y riesgo. El proyecto, además, supone una oportunidad para el análisis del impacto de las políticas sociales en la ciudadanía.

Palabras clave: segmentación; valoración social; diagnóstico social; riesgo social; servicios sociales.

\section{[en] DS-DIBA: An experience of producing a social assessment and segmentation tool for social services}

\begin{abstract}
One of the main challenges for basic primary care social services is improving the effectiveness and efficiency of the care process through segmentation. A redesign and continuous improvement research-action project was carried out across 16 municipalities in the province of Barcelona in order to make progress with this objective.

The segmentation procedure is implemented via DS-DIBA, a social assessment instrument that offers confidence, security and robustness in social diagnoses.

The main outcome is the identification by professional experts of the personal and family situations causing the needs that are the subject of social services intervention, in addition to the contextual factors that have an impact. This tool describes each situation based on objectifiable evidence and classifies it according to severity and frequency. Finally, each case is segmented under one of three risk levels depending on its impact for the cohabitation unit.

The implementation of DS-DIBA offers value as a support mechanism during the social intervention process. Common profiles can be identified among the user population, there is the scope for advances in share and structure language and in horizontal and vertical integration, cases can be more easily targeted, and interventions can be prioritised in terms of care intensity and risk. The project also represents an opportunity to analyse the impact of social policies on citizens.
\end{abstract}

Key words: segmentation; social assessment; social diagnosis; social risk; social services.

Servei d'Acció Social.Diputació de Barcelona, España manzanorm@diba.cat

2 Servei d'Acció Social. Diputació de Barcelona, España pagesge@diba.cat

3 Servei d'Acció Social. Diputació de Barcelona, España

solepmr@diba.cat 
Sumario: Introducción. 1. El diagnóstico social y la valoración social. 2. Las herramientas de valoración social. 3. Metodología. 4. Resultados. 4.1 Situaciones sociales. 4.2 Factores de contexto. 4.3 La determinación del riesgo. 4.4 Las necesidades sociales. 4.5 El nivel de riesgo y la segmentación. 4.6 La relevancia de la información. 4.7 Criterios de uso del instrumento de valoración social. 5. Conclusiones. 6. Referencias bibliográficas.

Cómo citar: Manzano Rodríguez, M.Á.; Pagés Gaulier, É.; Solé Pascual, M. (2019). La DS-DIBA: Experiencia de la elaboración de una herramienta de valoración social y segmentación para los servicios sociales, en Cuad. trab. soc. 32(1), 177-189.

\section{Introducción}

El papel y la eficacia de los servicios sociales básicos en Cataluña y en el Estado español han estado, durante los últimos años, en el centro del debate académico e incluso político. Su evolución histórica desde la beneficencia pública y la presión social a causa de la crisis económica, al centrarse en la gestión de prestaciones económicas de subsistencia de tipo asistencial (Aguilar-Hendrickson, 2013) ha llevado a una indefinición de su objeto de actuación. La consecuencia es un cierto "estancamiento" del desarrollo de la labor de prevención, protección y asistencia en el resto de sus dimensiones: la autonomía funcional y la interacción social. (Fantova, 2016).

Los servicios sociales afrontan este nuevo escenario con unos recursos en conocimiento y tecnología que datan del tiempo de su creación como sistema público de atención. A ello hay que sumar el bajo interés de la academia y de disciplinas afines por su modernización conceptual. Al debate existencial sobre su objeto y misión debe añadirse que los servicios sociales disponen de recursos limitados respecto al volumen de necesidades a cubrir, aspecto que, unido a una falta de tecnificación y sistematización de la intervención social, dificulta la fijación de prioridades, asignación eficiente de recursos y una atención coherente y equitativa. La consecuencia es que como sistema se identifican importantes dificultades para valorar y discriminar, de forma objetiva y sistematizada, las situaciones a atender y las necesidades sociales a cubrir, así como la prioridad e intensidad de la atención social.

\section{El diagnóstico social y la valoración social}

El diagnóstico social representa el elemento central del proceso de atención en los servicios sociales básicos. Tradicionalmente la valoración social en el sistema de servicios sociales se ha basado en las técnicas de prospección y diagnóstico del Trabajo Social que permiten una compresión general del caso. Sin embargo se observan carencias en la prioridad de los casos así como en el cribado de acceso a las prestaciones y los servicios del sistema. En términos más generales, se observa también una débil eficacia en la estructuración del proceso de intervención social e impacto de la actuación en la ciudadanía.

Actualmente la práctica profesional basa el diagnóstico de caso en la descripción de las características vitales de la persona-unidad de convivencia y en la enumeración de sus "problemáticas", entendidas como aquellas situaciones que afectan al bienestar de la persona (Ferrer y Montagud, 2012); resultando insuficiciente para conseguir el objetivo del diagnóstico social consistente en facilitar una comprensión holística del caso por el/la profesional (García, 2008; Díaz y De Castro, 2013).

Esta posición e individualización del caso a través del diagnóstico social no está permitiendo obtener una sistematización de la información que oriente a un resultado (García, 2008) en forma de valoración social. La carencia descrita plantea dificultades para determinar el grado de atención que recibirá la persona usuaria; las prestaciones y servicios asociados y, en general, la actuación que han de realizar los equipos profesionales. Todo ello resalta la ausencia de una modelización de la intervención social que plantee combinaciones en torno a intensidad de atención, servicios, prestaciones $\mathrm{y}$ actuaciones profesionales asociadas.

La causa principal es que las situaciones sociales problemáticas no han sido traducidas a una serie de variables estandarizadas, consensuadas y validadas de acuerdo a su importancia causal frente a la situación de necesidad y de gravedad en términos de riesgo social. El motivo es la complejidad de las situaciones-problema abordadas por los servicios sociales (Ferrer y Montagud, 2012); que son de característica multidimensional, dinámica y de intensidad diversa (Raya Díez, 
2007 y 2010), con vínculos que contienen información oculta al observador/a (Ferrer y Montagud, 2012).

A pesar de todo lo expuesto, los servicios sociales no pueden renunciar a un modelo de diagnóstico social "como proceso que sintetiza, interpreta y conceptualiza la naturaleza y magnitud de las necesidades sociales en sus efectos, génesis y causas personales y sociales", de acuerdo a la definición de Díaz y De Castro (2013, p. 433).

Esta tesis entiende la necesidad social como objeto y faro de la intervención social, siendo el diagnóstico una interpretación que va más allá de una sistematización de la información para avanzar hacía una adecuada valoración del caso entendida como "una interpretación de la situación de necesidad existente en comparación con un modelo ideal de referencia" (p.439).

Los servicios sociales necesitan, por lo tanto, dotarse de un método sistematizado de diagnóstico social - en el marco del proceso de intervención social con las personas usuarias-y disponer, a su vez, de instrumentos de valoración social que permitan "una determinada calificación, bien para el acceso a una prestación o servicio, o bien para fundamentar una decisión de carácter profesional" (García, 2008, p.14).

\section{Las herramientas de valoración social}

Actualmente no se dispone de un instrumento de diagnóstico o valoración social estandarizado y transferible con facilidad a la práctica de los servicios sociales en Cataluña. Así lo constata el reciente análisis efectuado por Avedis Donabedian (Ballester et al., 2016), a partir de una extensa revisión bibliográfica y consulta a expertos del sector.

Entre los instrumentos existentes se apuntan: la escala Gijón (García-González et al. 1999) dirigida al colectivo de ancianos y basada en variables y factores sociales descritos genéricamente sin profundizar en la interrelación y dependencia de las mismas; y que no prevé los factores de protección o riesgo que interaccionan con la necesidad social a cubrir; el instrumento de valoración de la exclusión social del País Vasco (Departamento de Empleo y Asuntos Sociales, 2012), centrado en el ámbito de la exclusión, siendo de compleja y costosa utilización como demestra su baja implantación (Orbegozo, 2016); o también herramientas de escala internacional, como el Self-Sufficiency Matrix en su adaptación holandesa, SSM-D (Lauriks et al., 2013); y el Northern Ireland Single Assessment Tool-NISAT (McCormack, Taylor, McConville, Slater y Murray, 2008) que, si bien aportan una completa relación de factores y aspectos clave para el diagnóstico social con algoritmos y escalas para favorecer la decisión,, en ambos casos se parte de una orientación y definición conceptual ajena cultural e organizativamente a la práctica de la intervención social en Cataluña.

Este contexto de experiencias, poco adaptables a la realidad de los servicios sociales catalanes, es el que justifica la necesidad de crear un instrumento de valoración social propio, con el objetivo de: personalizar la atención; disminuir la discrecionalidad; mejorar el contínuum asistencial de la persona usuaria; favorecer la equidad y mejorar la eficiencia del Sistema catalán de Servicios Sociales, en su nivel básico. La DS-DIBA es la propuesta de la Administración local al Sistema con la voluntad de completar el conocimiento generado por la Universidad y centros de investigación.

La DS-DIBA pretende cubrir la necesidad de segmentación y cribado en las diferentes fases del proceso asistencial, especialmente en acogida e intervención. La herramienta permite discriminar entre situaciones sociales y su gravedad e intensidad, con el objeto de diseñar planes personalizados de intervención y atención: intervenciones puntuales con un seguimiento profesional no intensivo para grupos de población en bajo riesgo o un mayor refuerzo con apoyos continuados y esenciales para los casos más complejos.

Asimismo, la creación de este instrumento de valoración social es esencial para diseñar procesos de atención integrada con otros sistemas de protección — salud, educación, ocupación, etc. - avanzado en el desarrollo de un lenguaje estandarizado y compartido dentro del sector.

\section{Metodología}

La experiencia descrita es el resultado de una metodología de investigación-acción (Lewin, 1973), que nace en el contexto de los proyectos de rediseño y mejora continua de los procesos de atención en los servicios sociales básicos de 16 municipios de la provincia de Barcelona. 
Estos municipios solicitan participar en el proyecto después de evaluar como organización la necesidad de iniciar un proceso de cambio y mejora continua (Murillo y Krichesky, 2012), siendo el único requisito de selección el compromiso explícito de participación realizado por sus responsables.

Durante la primera fase de creación de la herramienta DS-DIBA, se optó por la confección de una muestra no probabilística con representatividad de los diferentes tipos de estratos relacionados con el objeto de intervención: los disitintos perfiles profesionales que realizan el diagnóstico social (trabajadores sociales y educadores sociales) y los tamaños de los municipios (mayores y menores de 20.000 habitantes), ya que presentan modelos oganizativos y competenciales diferenciados.

La metodología propuesta se basa en la creación de conocimiento colaborativo con la participación de más de 200 profesionales de los equipos municipales de servicios sociales. Consideramos los equipos profesionales como expertos que con su juicio crean y validan el resultado de la investigación (Cabero y Llorente-Cejudo, 2013). Esta metodología cualitativa aporta valor por la calidad y profundidad de las contribuciones, así como el conocimiento profesional sobre contenidos difíciles, complejos y especializados, como son los de la intervención social, y permite, a la vez, establecer las bases de una futura validación estadística de la herramienta. La participación de profesionales en cada municipio se estructura en dos niveles:

1. Un grupo motor del proyecto: formado por entre 4 y 6 profesionales por municipio. $\mathrm{Su}$ selección se realiza con criterios de perfil profesional, trayectoria y motivación.

2. Un grupo de validación: formado por el conjunto de profesionales de cada municipio.

El proceso de trabajo se inició con sesiones de análisis de caso, con el objeto de identificar los elementos esenciales para el diagnóstico social, así como los factores que incidían en la valoración de la gravedad e importancia de las necesidades sociales. Los elementos identificados se clasificaron en un mapa conceptual y se definieron de forma consensuada en cada equipo de trabajo. Los resultados fueron validados en una sesión posterior. Los casos los aportaron los propios profesionales, a partir de una serie de premisas, con el fin de contemplar sus distintas tipologías e intervencions (gente mayor, infancia, casos nuevos, casos con alta cornicidad, etc.). Los casos se recopilaron en una ficha descriptiva y se han utilizado en diferentes municipios participantes en el estudio.

En una fase posterior, se determinaron los niveles de gravedad de cada situación social relevante, la identificación de evidencias que permitían discriminar entre un nivel u otro; la concordancia de aquellos con incidencia en el aumento o disminución del nivel de gravedad $\mathrm{y}$, finalmente, la ponderación de la misma para cada ámbito de necesidad, con el objetivo de discriminar el nivel de riesgo global de la persona o unidad de convivencia analizada. Este proceso comprendió varias sesiones de validación. En cada uno de los grupos motor se revisaba la adecuación del instrumento de valoración a la diferente tipología de situaciones de necesidad social y niveles de gravedad.

En el proceso de validación se siguió la metodología propuesta por Maxwell y Stake (2006), asegurando un amplio consenso (más del 80 por ciento de profesionales) en la descripción y definición de las categorias y situaciones (validez descriptiva), en el encaje de la propuesta teórica (validez teórica), en el ajuste de los distintos niveles de gravedad con cada una de las situaciones propuestas (validez interpretativa) $\mathrm{y}$, finalmente, en la aceptación que el grado de riesgo propuesto es generalizable al conjunto de casos con situaciones análogas (generalidad). Todo el proceso se replicó en los equipos de los 16 municipios participantes. El conocimiento generado en cada grupo se incorporó al instrumento de diagnóstico social en un trabajo de consolidación y validación colectiva. Durante los cuatro años de desarrollo de la experiencia se han realizado un total de 72 sesiones de estudio de casos y 35 sesiones de validación.

Para establecer los criterios de ponderación y determinación de los niveles de riesgo se realizaron - una vez concluida la validación individual de cada una de las categorías-, cuatro sesiones de trabajo de un grupo de expertos, con el fin de categorizar el peso de cada una de las situaciones y de los ámbitos en la valoración final del riesgo global del caso, a partir de la utilización del método estadístico de análisis conjunto (modelo composicional multiatributo). 


\section{Resultados}

El resultado es un instrumento de valoración social (Figura 1), estructurado en los tres ám- bitos de necesidad social definidos en el Plan Estratégico de Servicios Sociales de Cataluña 2010-14 (Maluquer, Fustier y Recio, 2010).

Figura 1: Mapa conceptual instrumento de diagnóstico social.

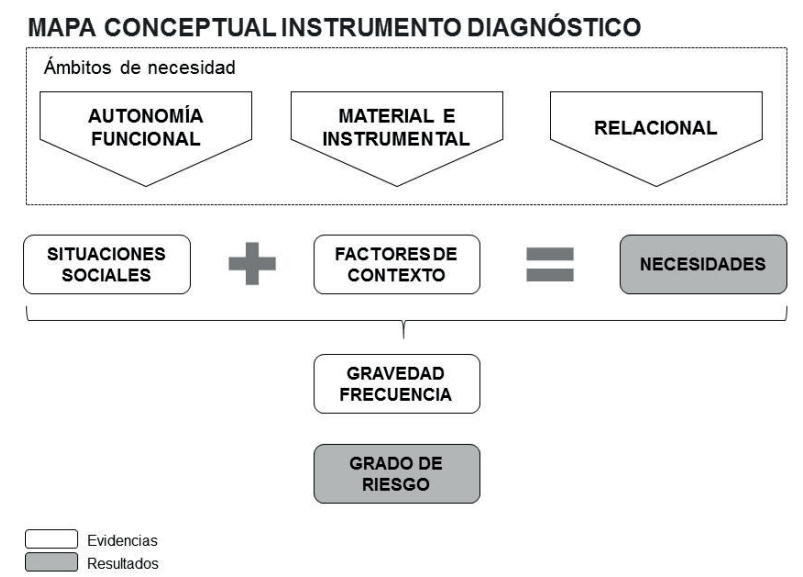

Fuente: Elaboración propia.

La elección de esta clasificación fue, en un primer momento, práctica. Las fuentes estudiadas proponían modelos poco adaptados a la operativa de los servicios sociales básicos en Cataluña y resultaban excesivamente largos y complejos. Posteriormente se ha valorado que esta clasificación orienta convenientemente el instrumento a la obtención de una valoración social a conceptualizar y evaluar las necesidades sociales a atender (Díaz y De Castro, 2013)

Los tres ámbitos de necesidad se definen de la siguiente forma:

- Autonomía funcional: estado permanente en que se encuentran las personas que, por razones derivadas de la edad, la enfermedad o la discapacidad, y vinculadas a la falta de autonomía física, mental, intelectual o sensorial, precisan atención de otras personas o ayudas importantes para realizar las actividades básicas de la vida diaria; o en el caso de las personas con discapacidad intelectual o enfermedad mental, otros apoyos instrumentales para mantener su autonomía personal.
- Relacional: Situaciones de necesidad derivadas de los vínculos sociales, tanto en el entorno familiar como en el comunitario. En esta categoría se incluyen todas las situaciones de necesidad en las que el déficit en las relaciones sociales, la falta de relaciones y/o la existencia de unas relaciones disfuncionales suponen un riesgo en el desarrollo psicosocial de la persona.

- Material e instrumental: Situaciones de necesidad que tienen una expresión de tipo material, vinculadas con la subsistencia y la capacidad de las personas para alcanzar esta subsistencia de forma autónoma.

Los ámbitos de necesidad se distribuyen en 7 entornos de intervención (Cuadro 1) por parte de los servicios sociales. La clasificación del entorno es meramente funcional y tiene como objeto ayudar a los y las profesionales en el proceso de identificación de las situaciones sociales. En la práctica se ha demostrado la utilidad de los entornos en la búsqueda de relaciones y causalidades entre las situaciones sociales y los factores de protección. 
Cuadro 1: Entornos de intervención

\begin{tabular}{cc}
\hline Tipo Necesidad & Entorno \\
\hline Autonomía funcional & Vida diaria e instrumental \\
Material e instrumental & Vivienda \\
& Económica \\
Relacional & Laboral \\
& Escolar \\
& Familiar \\
\hline
\end{tabular}

Fuente: Elaboración propia

Existe una cierta contradicción entre la delimitación del perímetro de los servicios sociales realizada por Fantova, citado anteriormente, y la propuesta de ámbitos de necesidad y entornos que propone la herramienta. Y es que si bien DS-DIBA coincide en que el objeto, ha de centrarse en la interacción de la autonomía funcional e integración relacional, se entiende que el instrumento debe abarcar y ser sensible a los ámbitos de actuación actuales de los equipos profesionales en su rol de facilitación y apoyo para el acceso a servicios y prestaciones de otros sistemas, acompañando a las personas en el ejercicio de sus derechos sociales.

\subsection{Situaciones sociales}

En cada entorno se identifican situaciones sociales en las que se pueden encontrar las perso- nas objeto de atención o sus unidades de convivencia y que pueden ser consideradas como un problema para su bienestar (Ferrer y Montagud, 2012). Se ha realizado un esfuerzo para no incluir aquellas necesidades que son objeto prioritario de otros sistemas de bienestar. La descripción de cada situación social se ha acotado a partir de la identificación, mediante la observación y el análisis de caso, de unos hechos probados (evidencias objetivas y contrastadas). La identificación de estas situaciones permite determinar las necesidades sociales de la persona o de la familia a atender, en función de la misión y cartera de los servicios sociales básicos. Representan la existencia de una discrepancia entre el estado actual y el estado deseado.

Se han identificado y definido 31 situaciones sociales (Cuadro 2) susceptibles de intervención por parte de los servicios sociales básicos ${ }^{4}$.

Cuadro 2: Listado situaciones sociales

\begin{tabular}{|c|c|c|}
\hline \multicolumn{3}{|l|}{ Situaciones sociales } \\
\hline Autonomía funcional & Material e instrumental & Relacional \\
\hline $\begin{array}{l}\text { - Falta de autonomía personal en las actividades } \\
\text { básicas de la vida diaria } \\
\text { - Falta de autonomía personal en las actividades } \\
\text { instrumentales de la vida diaria }\end{array}$ & $\begin{array}{l}\text { - Falta de vivienda } \\
\text { - Vivienda deficiente } \\
\text { - Vivienda insegura } \\
\text { - Vivienda masificada } \\
\text { - Riesgo de pérdida o falta de servicios / sumi- } \\
\text { nistros } \\
\text { - Sin ingresos } \\
\text { - Ingresos insuficientes } \\
\text { - } \text { Mala administración } \\
\text { - Falta de calificación y/o orientación laboral } \\
\text { sales competencias profesionales transver- }\end{array}$ & 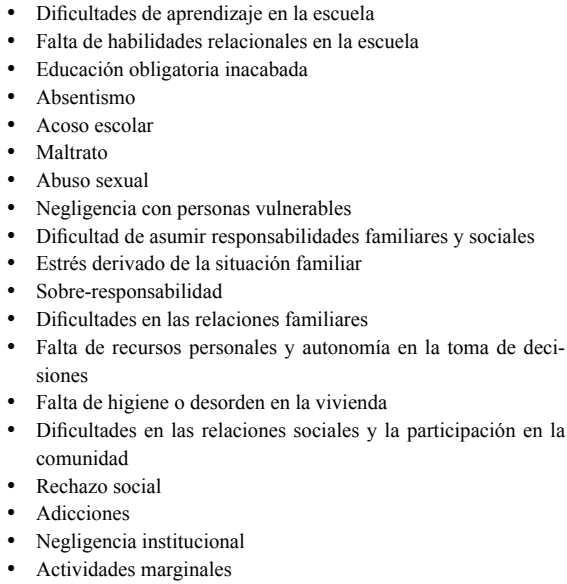 \\
\hline
\end{tabular}

Fuente: Elaboración propia

Las tablas con la descripción de cada situación social se pueden descargar en este enlace: http://www.diba.cat/documents/14465/70049014/Anexo+tablas+instrumento+valoraci\%C3\%B3n+social+DS-DIBA.pdf/99ff20b4-b9d1-4db5-8b32d84ea22cea86 
La aplicación operativa del instrumento de valoración se estructura en la identificación del tipo de necesidad relacionada con la situación social que presenta una mayor gravedad. Se indica qué situaciones son relevantes y se determina la necesidad de intervención social. No se ha establecido un límite máximo en el número de situaciones a valorar; pero operativamente se consideran difícilmente abordables más de 5 situaciones simultáneamente.

Cada una de las situaciones sociales puede afectar a uno o diversos miembros de la unidad de convivencia. El instrumento permite discriminar este factor con objeto de realizar una ponderación del riesgo correcta y la orientación de la intervención social.

La herramienta admite la identificación de otras situaciones sociales que afectan a la persona o a la familia, con la finalidad de conocer y describir el caso de una forma pormenorizada, pero que no derivan en una necesidad social y, por lo tanto, no son objeto de una intervención social de los servicios sociales. El instrumento recomienda que se relacionen en el apartado de observaciones con la finalidad de poder recuperarlas en reevaluaciones posteriores, o con el propósito de hacer más comprensible el caso.

\subsection{Factores de contexto}

La teoría sistémica del Trabajo Social ha conceptualizado, hace ya tiempo, a la persona objeto de intervención social como miembro de un sistema que no puede ser entendido de forma separada de su entorno y de las relaciones que se construyen (Ferrer y Montagud, 2012). También el modelo ecológico ha determinado que hay factores de riesgo y de protección, presentes en los distintos niveles que influyen en la situación social (Cicchetti y Rizley, 1981, citado en Rodríguez, Camacho, Rodrigo, Martín y Máiquez, 2006)

Se han identificado 43 factores de contexto, entendidos como aquellas características del individuo o de su familia; de su posicionamiento, de su contexto y entorno, que pueden contribuir a aumentar, inhibir, reducir o atenuar el riesgo que presenta el individuo o familia. Los factores de contexto pueden ser relevantes en relación a todo el caso (Cuadro 3) o a una de las dimensiones de necesidad. Estos factores se consideran de riesgo en función de su relevancia, a criterio profesional, en el aumento de la gravedad de la situación o riesgo del caso. En la conyuntura que el efecto relevante conlleve la disminución de la gravedad de la situación o riesgo, se consideran factores de protección.

Cuadro 3: Factores de contexto globalidad del caso

\section{GLOBALIDAD DEL CASO}

Factores de protección

- La persona y/o familia tiene conciencia y acepta la dificultad/enfermedad causante de la situación

- La persona y/o familia acepta el apoyo y la orientación profesional.

- La persona conoce y tiene una actitud proactiva y participativa hacia el sistema y las redes de protección.

\section{Factores de riesgo}

- La persona y/o familia no tiene conciencia y/o no acepta la enfermedad/dificultad causante de su situación.

- La persona y/o familia no acepta el apoyo y la orientación profesional.

- La persona no tiene una actitud activa ni participativa hacia los sistemas y redes de protección y/o tiene un posicionamiento victimista respecto a ellos.

- Composición familiar vulnerable (monoparental, familias numerosas de categoría especial, etc.) 
En el Cuadro 4 se relacionan los factores de contexto en relación a las dimensiones de necesidad.

Cuadro 4: Factores de contexto según necesidades

\begin{tabular}{|c|c|c|c|c|c|}
\hline \multicolumn{2}{|c|}{ NECESIDADES AUTONOMÍA FUNCIONAL } & \multicolumn{2}{|c|}{$\begin{array}{l}\text { NECESIDADES MATERIALES E } \\
\text { INSTRUMENTALES }\end{array}$} & \multicolumn{2}{|c|}{ NECESIDADES RELACIONALES } \\
\hline $\begin{array}{l}\text { Factores de pro- } \\
\text { tección }\end{array}$ & Factores de riesgo & $\begin{array}{l}\text { Factores de pro- } \\
\text { tección }\end{array}$ & Factores de riesgo & $\begin{array}{l}\text { Factores de pro- } \\
\text { tección }\end{array}$ & Factores de riesgo \\
\hline $\begin{array}{l}\text { La enfermedad } \\
\text { causante del déficit } \\
\text { de autonomía está } \\
\text { diagnosticada y en } \\
\text { tratamiento } \\
\text { - La persona tiene re- } \\
\text { conocidos el grado } \\
\text { de discapacidad y / o } \\
\text { dependencia } \\
\text { - La persona es usu- } \\
\text { aria de un servicio, } \\
\text { recurso o prestación } \\
\text { en función de su en- } \\
\text { fermedad, discapa- } \\
\text { cidad o situación de } \\
\text { dependencia } \\
\text { Hay una red familiar } \\
\text { y / o social de apoyo } \\
\text { implicada en el cui- } \\
\text { dado } \\
\text { La persona sigue } \\
\text { con regularidad el } \\
\text { tratamiento médico } \\
\text { y / o tiene un hábitos } \\
\text { saludables por su si- } \\
\text { tuación de salud }\end{array}$ & $\begin{array}{l}\text { - Se prevé que el déficit } \\
\text { de autonomía de la } \\
\text { persona se agrave en } \\
\text { un corto plazo (durante } \\
\text { el año) } \\
\text { - La enfermedad causan- } \\
\text { te del déficit de autono- } \\
\text { mía no está diagnosti- } \\
\text { cada y en tratamiento } \\
\text { - La persona no sigue } \\
\text { con regularidad el tra- } \\
\text { tamiento médico pres- } \\
\text { crito y/o no tiene unos } \\
\text { hábitos saludables por } \\
\text { su situación de salud } \\
\text { - No existe una red fami- } \\
\text { liar y / o social que se } \\
\text { implique en el cuidado } \\
\text { Cuidador/a habitual en } \\
\text { riesgo o en situación de } \\
\text { claudicación } \\
\text { La situación y / o ca- } \\
\text { racterísticas del domi- } \\
\text { cilio agravan el déficit } \\
\text { de autonomía de las } \\
\text { personas que viven } \\
\text { La persona con déficit } \\
\text { de autonomía tiene res- } \\
\text { ponsabilidades / cargas } \\
\text { familiares }\end{array}$ & $\begin{array}{l}\text { - Una o más personas } \\
\text { de la familia dispone } \\
\text { de ingresos estables } \\
\text { (pensiones, presta- } \\
\text { ciones, rentas, etc.). } \\
\text { - Una o más personas } \\
\text { de la familia dispone } \\
\text { de ahorros } \\
\text { - Una o más personas } \\
\text { de la familia dispone } \\
\text { de patrimonio aparte } \\
\text { de la primera resi- } \\
\text { dencia } \\
\text { - Una o más personas } \\
\text { de la familia dispone } \\
\text { de trabajo estable. } \\
\text { Existe red familiar o } \\
\text { relacional dispuesta } \\
\text { a ofrecer apoyo eco- } \\
\text { nómico } \\
\text { En caso de separa- } \\
\text { ción o divorcio hay } \\
\text { convenio regulador } \\
\text { y se cumplen sus } \\
\text { términos } \\
\text { La persona tiene una } \\
\text { buena predisposi- } \\
\text { ción para formarse y } \\
\text { / o trabajar } \\
\text { Se dispone de una } \\
\text { vivienda habitual } \\
\text { por la que no se debe } \\
\text { asumir ningún gasto } \\
\text { o es una vivienda } \\
\text { social }\end{array}$ & $\begin{array}{l}\text { - Existe un impago } \\
\text { de pensiones de } \\
\text { manutención y la } \\
\text { correspondiente } \\
\text { demanda judicial } \\
\text { - Existen responsa- } \\
\text { bilidades y cargas } \\
\text { familiares que } \\
\text { agravan la falta de } \\
\text { recursos materiales } \\
\text { /instrumentales } \\
\text { - Hay un endeuda- } \\
\text { miento creciente e } \\
\text { inalcanzable con } \\
\text { los recursos dispo- } \\
\text { nibles }\end{array}$ & $\begin{array}{l}\text { - La persona y/o familia } \\
\text { muestra una vincula- } \\
\text { ción relacional positiva } \\
\text { con la familia extensa } \\
\text { o sus redes de amigos y } \\
\text { relaciones } \\
\text { - La persona está vincu- } \\
\text { lada y participa activa- } \\
\text { mente con la comuni- } \\
\text { dad, entidades o asoci- } \\
\text { aciones de su entorno } \\
\text { - Existe red familiar o } \\
\text { relacional dispuesta a } \\
\text { ofrecer apoyo y acom- } \\
\text { pañamiento } \\
\text { - En el caso de infancia } \\
\text { que el niño/a mantiene } \\
\text { relación afectiva con } \\
\text { adultos de referencia e } \\
\text { iguales } \\
\text { - En el caso de infancia } \\
\text { que el niño/a muestra } \\
\text { una autonomía personal } \\
\text { adecuada para su edad } \\
\text { En el caso de infancia } \\
\text { que el niño/a participa } \\
\text { en actividades fuera } \\
\text { del entorno familiar o } \\
\text { escolar } \\
\text { La persona / familia } \\
\text { es usuaria de recursos } \\
\text { / servicios dirigidos a } \\
\text { cubrir la necesidad en } \\
\text { el ámbito relacional } \\
\text { (escolar, familiar o co- } \\
\text { munitario) }\end{array}$ & $\begin{array}{l}\text { - Existe una red social (veci- } \\
\text { nos/as, amigos/as, familia } \\
\text { extensa,...) que ejerce una } \\
\text { influencia negativa } \\
\text { - Sospecha o diagnóstico } \\
\text { de enfermedad mental que } \\
\text { agravan la situación } \\
\text { - Existe una problemática de } \\
\text { salud no diagnosticada que } \\
\text { agrava la situación } \\
\text { - En el caso de infancia que } \\
\text { el niño/a no mantiene re- } \\
\text { lación afectiva con adultos } \\
\text { de referencia e iguales } \\
\text { - En el caso de infancia que } \\
\text { el niño/a no muestra una } \\
\text { autonomía personal adecu- } \\
\text { ada para su edad } \\
\text { En el caso de infancia que } \\
\text { el niño/a no participa en ac- } \\
\text { tividades fuera del entorno } \\
\text { familiar o escolar }\end{array}$ \\
\hline
\end{tabular}

Fuente: Elaboración propia

\subsection{La determinación del riesgo}

Se han establecido tres niveles de riesgo para cada una de las situaciones sociales incluidas en el instrumento de diagnóstico: baja, moderada y alta.

El nivel de riesgo de cada situación es el resultado de la ponderación de dos factores: gravedad y frecuencia, en la observación de la misma.

La gravedad es la valoración de la intensidad de la situación en base a las consecuencias o daño que puede provocar en la persona o familia. Se divide en tres niveles: baja, moderada o alta. En algunas situaciones se conside- ran solo los niveles de gravedad más altos. La determinación de la gravedad está basada en una o más evidencias que permiten discriminar entre los tres niveles.

La frecuencia es la repetición reiterada y en intervalos de la situación. Se le asignan los valores de ocasional, frecuente o continua, en función de su periodicidad o número de repeticiones, siempre con relación al tipo de situación que se trata. Para cada situación, se han definido las evidencias que permiten discriminar entre los distintos niveles. En determinadas situaciones no se valora la frecuencia ya que se considera irrelevante. ${ }^{4}$

\footnotetext{
Las tablas con las definiciones de la gravedad y frecuencia de cada situación y las evidencias para la observación se pueden descargar en el siguiente enlace: http:/www.diba.cat/documents/14465/70049014/Anexo+tablas+instrumento+valoraci\%C3\%B3n+social+DS-DIBA.pdf/99ff20b4-b9d1-4db5-8b32-d84ea22cea86
} 


\subsection{Las necesidades sociales}

El instrumento de valoración social es el fundamento de un modelo de intervención centrado en la persona. Permite establecer la prioridad y pertinencia de las actuaciones, así como las prestaciones sociales prescritas con el objetivo de incidir sobre las situaciones sociales detectadas.

En este punto es necesario detenerse en el concepto de necesidad social básica. Tal y como señalan Díaz y De Castro: "hace referencia a los factores objetivos indispensables para el mantenimiento de la vida y la reproducción social" son "de naturaleza y origen sociales y su satisfacción únicamente puede producirse en la interacción y relación social" (2013, p.440).

La necesidad social, en el marco de la herramienta de valoración, se considera como una carencia que ha de ser objeto de una intervención social (García, 2008), relevante y factible del sistema de servicios sociales. Viene motivada por una o más situaciones sociales. El tratamiento de la necesidad es fundamental para la delimitación de los objetivos del plan de intervención y para la acción profesional.

Cuadro 5: Necesidades sociales

\begin{tabular}{|c|c|c|}
\hline \multicolumn{3}{|l|}{ Necesidades sociales } \\
\hline Autonomía funcional & Materiales e instrumentales & Relacionales \\
\hline $\begin{array}{l}\text { - Apoyo a la alimentación. } \\
\text { - Apoyo a la higiene. } \\
\text { - Apoyo al seguimiento y tratamiento médico } \\
\text { y control farmacológico. } \\
\text { - Apoyo a la protección de bienes y patri- } \\
\text { monio. } \\
\text { - Apoyo al bienestar emocional. }\end{array}$ & $\begin{array}{l}\text { - Alojamiento temporal y para hacer } \\
\text { frente a situaciones sobrevenidas. } \\
\text { - Acceso a una vivienda. } \\
\text { - Acondicionamiento de la vivienda. } \\
\text { - Cobertura de las necesidades básicas } \\
\text { (alimentación, suministros, ropa, etc.). } \\
\text { - Apoyo a la gestión de la economía } \\
\text { familiar. } \\
\text { - Mejora de las condiciones socio labo- } \\
\text { rales y / o de la empleabilidad para la } \\
\text { inserción laboral. }\end{array}$ & $\begin{array}{l}\text { - Protección física, psicológica, jurídica, etc. } \\
\text { - Adquisición de patrones de conducta socialmente establecidos. } \\
\text { - Adquisición de dinámicas y roles familiares socialmente establecidos. } \\
\text { - Promoción / empoderamiento individual / adquisición de habilidades y } \\
\text { recursos personales. } \\
\text { - Apoyo para poder afrontar las cargas familiares. } \\
\text { - Atención y cuidado. } \\
\text { - Apoyo a la mejora de las relaciones sociales y a la inclusión social. } \\
\text { - Recibir tratamiento médico y / o terapéutico. }\end{array}$ \\
\hline
\end{tabular}

Fuente: Elaboración propia

\subsection{El nivel de riesgo y la segmentación}

El conjunto de situaciones sociales, su valoración y los factores de contexto, determinan el diagnóstico de cada ámbito de necesidad y su clasificación de riesgo en base a los tres niveles propuestos: vulnerable, de riesgo o de alto riesgo. El caso tendrá una valoración en función del riesgo determinado en cada uno de los tres ámbitos de necesidad y de la incidencia de los factores de contexto globales del caso.

En la fase actual de desarrollo de la herramienta, el riesgo de los ámbitos de necesidad y caso se establece a criterio profesional, en función de la información disponible. Por lo tanto, es este juicio el que pondera la importancia de cada ámbito y determina el riesgo global del caso. Generalmente, la calificación de cada caso será el riesgo más alto de cualquiera de los ámbitos de necesidad. Afirmación limitada por la ponderación profesional a la baja de las necesidades de tipo material e instrumental y por la incidencia de los factores de protección globales al caso.

\subsection{La relevancia de la información}

La DS-DIBA es un instrumento de valoración del caso teniendo, como misión principal, su clasificación en niveles de riesgo. La herramienta ofrece además valores complementarios a destacar:

- Permite especificar las situaciones sociales y los factores de contexto para los miembros de la unidad de convivencia, obteniendo valoraciones singularizadas.

- Identifica las situaciones sociales relevantes para la atención a las necesidades sociales y, por lo tanto, se posiciona como un instrumento esencial para la formulación de planes de intervención.

- Determina factores de protección que pueden actuar como palanca de cambio y objetivos de trabajo compartido con las personas usuarias.

- Ofrece una panorámica de las necesidades sociales presentes en un territorio, aspecto 
clave en la definición de políticas y focalización de recursos: ecómicos, técnicos y tecnológicos.

En el marco de la atención de los servicios sociales básicos, DS-DIBA no puede ni debe entenderse como el único de los instrumentos de valoración social a utilizar. Su misión es la segmentación de casos y la de actuar como base diagnóstica para la formulación de planes de intervención social generales.

Algunas prestaciones y actuaciones especializadas desempeñadas por los servicios sociales básicos necesitaran dotarse de otros instumentos específicos o complementarios que profundicen y focalizen la valoración social. Ejemplo de ello serían las escalas de calidad de vida en la promoción de la autonomía o de riesgo psicosocial, en el caso de sospecha de maltrato infantil.

\subsection{Criterios de uso del instrumento de valoración social}

La DS-DIBA es un instrumento de valoración social diseñado para su utilización en todas las fases del continuum asistencial de los servicios sociales básicos. Asimismo se presenta como un complemento a las metodologías y procesos de trabajo de las respectivas disciplinas profesionales que conforman los equipos de trabajo.

La persona usuaria es atendida por el o la profesional en el marco de una entrevista ya sea individual, grupal o domiciliaria, donde se realizan las tareas de prospección y diálogo necesarias para la formulación del diagnóstico social. Es en la determinación de la valoración social de la situación, si fuera necesario, cuando el o la profesional pueden utilizar la DS-DIBA.

En el marco de la investigación se han definido, a escala local, estándares del número de actuaciones (entrevistas, visitas, etc.) deseables en cada atención; y se han identificado los momentos del proceso donde sería necesaria la valoración social, para definir la continuidad de la atención y/o la prescripción de alguna prestación o recurso.

La DS-DIBA es la síntesis valorativa de la exploración social global y no un cuestionario, tipo chek-list, a cumplimentar en todas las atenciones. La forma en que se utiliza el instrumento es relevante para el resultado final de la valoración. Se propone la siguiente secuencia:
1. Recopilación de hechos, evidencias y posicionamiento de la persona usuaria en el proceso general de la entrevista y/o visita a domicilio.

2. Identificación del ámbito de necesidad del que se sospecha, a priori, un mayor riesgo. Evaluación de las situaciones sociales relevantes, determinación de los miembros de la unidad de convivencia afectados y establecimiento de la gravedad y frecuencia. El resultado de esta valoración aporta un listado de situaciones y el riesgo asociado a cada una de ellas.

3. Decisión en torno a los factores de contexto (riesgo o protección) con incidencia sobre las situaciones sociales detectadas.

4. Confirmación del nivel de riesgo en el primer ámbito de necesidad analizado (se determina, a criterio profesional, mediante la ponderación del riesgo de cada situación social y de los factores de contexto).

5. Repetición de los pasos de 2 a 4, para los otros dos ámbitos de necesidad y obtención, de los niveles de riesgo en los tres ámbitos.

6. Determinación del nivel de riesgo global del caso, a partir del análisis (efectuado en base a criterio profesional) de los tres niveles de riesgo y factores de contexto que afectan globalmente al caso.

Apuntar que la DS-DIBA no es un instrumento estático para su uso exclusivo durante el proceso de diagnóstico inicial de acogida. Es un instrumento de valoración dinámico que desarrolla todo su potencial, cuando se inscribe en el continuum asistencial de los servicios sociales básicos. Se destaca así mism que es sensible a las variaciones y los cambios de la situación social, siendo recomendable su uso cuando el o la profesional tienen evidencias de los mismos.

También se recomienda su utilización en los procesos de evaluación y revisión del caso, ya sea con una temporalidad fija o a petición de nuevas demandas de la persona usuaria objeto de atención.

La herramienta nacida des del bottom up refuerza la organización a partir de una marcada vocación sistémica. La acumulación de evidencia generada por la intervención-acción amplía el conocimiento de las necesidades de la población y, a medio plazo, su impacto en la 
cartera de servicios y prestaciones así como en la calidad de vida de la ciudadanía. Ampliando, la capacidad de acción de las políticas sociales.

\section{Conclusiones}

La DS-DIBA es un instrumento de valoración social que permite avanzar en la estandarización de las situaciones sociales susceptibles de ser atendidas desde los servicios sociales básicos en Cataluña. Se enmarca en los procesos de atención y prácticas profesionales en el ámbito del diagnóstico social.

El principal logro es poder determinar un nivel de riesgo en cada caso, disponer de un listado de situaciones sociales susceptibles de intervención social y de los factores de contexto que inciden en su ponderación. El resultado es una ficha de caso analítica y sintética que completa el diagnóstico social inicial.

La investigación ha permitido determinar las necesidades sociales susceptibles de ser abordadas por el Sistema público y que deben de ser objeto de su actuación. Por otra parte, segmentarlas por tipología, gravedad y afectación al bienestar de la ciudadanía.

Asimismo se destaca que ha sido creado des del bottom up incidiendo des de la base en la mejora del sistema de servicios sociales. $\mathrm{Y}$ se apuntan las tres dimensiones, micro-meso-macro, y principales impactos derivados de la implantación de la herramienta de valoración y segmentación social.

- A nivel macro, intervención profesional, el instrumento garantiza una visión holística del caso y de las situaciones de necesidad asociadas; centrando la atención en los cambios de las situaciones sociales que pueden comportar un mayor riesgo para la persona usuaria. Permite también disponer de elementos objetivos para priorizar actuaciones y determinar la intensidad, según cada caso; acotando el marco de la intervención profesional y focalización del mismo; aportando seguridad y respaldo y; finalmente, facilitando y sistematizando el traspaso entre profesionales y unidades organizativas.

- A nivel meso, organizativo de los servicios sociales básicos, contribuye en la disminución de la variabilidad en las decisiones profesionales y su efecto de inequidad en las actuaciones, mediante la sistematización en la elaboración del diagnóstico y, por extensión, del plan de intervención. También es un instrumento para la planificación, prioridad y evaluación de las líneas estratégicas, los recursos y las prestaciones, en función de la prevalencia de las necesidades sociales detectadas.

- A nivel macro, sistema de servicios sociales, puede ser un instrumento para orientar la cartera de servicios y prestaciones; proporciona conocimiento para la planificación y distribución de los recursos, en función de la situación social de cada territorio, aportando así mismo evidencias para la evaluación.

En el campo de la intervención social se complementa con otras herramientas especializadas de cribado o valoración (infancia, autonomía, etc.), avanzando hacía un lenguaje compartido dentro del sector.

La creación de un instrumento de valoración social es el primer paso en la construcción de un modelo de intervención social orientado a la visión sistémica y centrado en la persona. En un futuro se deberá avanzar en la operatividad de los objetivos de intervención social planteados, la estandarización de las actuaciones, la prescripción de prestaciones así como una evaluación continuada y sistemática.

En el momento de redacción del presente artículo ya se trabaja en la confección de un algoritmo que permita la determinación del riesgo para cada ámbito de necesidad y del caso, en general, en función del itinerario en los servicios sociales básicos. La ponderación de la relevancia para el caso de cada situación social o factor de contexto, se está realizando mediante la metodología de consulta a personas expertas, utilizada ya en la construcción del instrumento.

Entre los retos de futuro de la herramienta, DS-DIBA, está la realización de una prueba piloto, a gran escala, para poder efectuar un análisis psicométrico de su consistencia interna, fiabilidad interámbitos y validez del constructo. Para el desarrollo de algoritmos y, si es necesario, de módulos para su uso en el acceso a servicios especializados. Esta prueba piloto y validación se realizará en cooperación con la Universidad, en un intento de fomentar la co- 
laboración entre los agentes implicados en la generación de conocimiento. Finalmente, para obtener un uso masivo dentro del sector, es vital mejorar la capacidad de las organizacions en la disposición de datos estructurados y sig- nificativos, mejorando su capacidad de análisis y evaluación. Por esta razón resulta esencial que se concluya el instrumento dentro de los principales sistemas de gestión e información de los servicios sociales básicos en Cataluña.

\section{Referencias bibliográficas}

Aguilar-Hendrickson, M.A. (2013). Los servicios sociales en la tormenta. Documentación social, 166, 145-167.

Aguilar Idáñez, Ma J. (2013). Trabajo social. Concepto y metodología. Madrid: Paraninfo y Consejo General del Trabajo Social.

Ballester, M., Hilarión, P., Domínguez, J.A., Delgado, X., Sarquella, E., de Andrés, J. y Sunol, R. (2016). Screening for Social Care Complex Needs - Review of current experiences. [Ponencia presentada en la 16th International Conference on Integrated Care. Barcelona: España, mayo].

Cabero, J. y Llorente-Cejudo, M.C. (2013). La aplicación del juicio de experto como técnica de evaluación de las tecnologías de la información (TIC). Eduweb, 7(2), 11-22.

Cardona Cardona, J., Cuartero Castañer, M.E. y Campos Vidal, J.F. (2016). La comprensión de la situación problema en la práctica del Trabajo Social de Casos. Cuadernos de Trabajo Social, 30(1), 149-162. doi: http://dx.doi.org/10.5209/CUTS.51735

Cicchetti, D. y Rizley, R. (1981). Developmental perspectives on the etiology, intergenerational transmission, and sequelae of child maltreatment. New Directions for Child and Adolescent Development, 1981(11), 31-55. doi: http://dx.doi.org/10.1002/cd.23219811104

Departamento de Empleo y Políticas Sociales. (2011). Balora. Instrumento para la valoración de la gravedad de las situaciones de riesgo y desamparo en los servicios sociales municipales y territoriales de atención y protección a la infancia y adolescencia en la Comunidad Autónoma Vasca. Vitoria-Gasteiz: Servicio Central de Publicaciones del Gobierno Vasco. Recuperado de:

http://www.euskadi.eus/contenidos/informacion/balora/es_doc/adjuntos/ACTUALIZACION_INSTRUMENTO_BALORA.bopv.pdf

Departamento de Empleo y Asuntos Sociales. (2012). Instrumento técnico común de valoración de la exclusión social. Ámbito convivencial. Vitoria-Gasteiz: Eusko Jaurlaritza-Gobierno Vasco. Recuperado de:

http://www.euskadi.eus/contenidos/informacion/instrumento_valoracion/es_instrume/adjuntos/Instrumento\%20valoracion\%20exclusion\%20social-junio\%202012.pdf.

Díaz Herráiz, E. y Fernández de Castro, P. (2013). Conceptualización del diagnóstico en Trabajo Social: necesidades sociales básicas Cuadernos de trabajo social, 26(2), 431. DOI: http://dx.doi.org/10.5209/ rev CUTS.2013.v26.n2.39550

Escobar-Pérez, J. y Cuervo-Martínez, A. (2008). Validez de contenido y juicio de expertos: una aproximación a su utilización. Avances en medición, 6(1), 27-36.

Fantova, F. (2016). Servicios Sociales y atención integrada. Actas Coordinación Sociosanitaria, 18, 7-19.

Ferrer, J. y Montagud, X. (2012). Trabajo social y complejidad: los "wicked problems. En: O. Vázquez Aguado y Y. de la Fuente (sds.), El trabajo social ante los desafios de un mundo en cambio (pp.10351052). Huelva: Servicio de Publicaciones de la Universidad de Huelva.

García, G.A. (2008). Reflexiones y utilidades sobre el diagnóstico y la programación de la intervención social. Ecos de Mary Richmond. Revista de Servicios Sociales y Politica Social, 83, 9-38.

García González, J.V., Díaz Palacios, E., Salamea García, A., Cabrera González, D., Menéndez Caicoya, A., Fernández Sánchez, A. y Acebal García, V. (1999). Evaluación de la fiabilidad y validez de una escala de valoración social en el anciano. Atención Primaria, 23(7) , 434-440.

Lauriks, S., Buster, M., De Wit, M., Van de Weerd, S., Theunissen, V., Schönenberger, M. y Fassaert, T. (2013). Manual and description of the Self Sufficiency Matrix 2013. Amsterdam: GGD. Recuperado de: http://www.selfsufficiencymatrix.org/zrm-int/publications/manual-to-the-ssm

Lewin, K. (1973). Actionresearchandminorityproblems, en Resolving Social Conflicts: Selected Papers on Group Dynamics. Londres: SouvenirPress 
Maluquer, E., Fustier, N. i Recio, M. (dirs.) (2010). Pla estratègic de Serveis Socials de Catalunya 20102013. Barcelona: Generalitat de Catalunya. Recuperat de http://treballiaferssocials.gencat.cat/ca/detalls/Article/Pla-estrategic-de-serveis-socials-de-Catalunya-2010-2013

Maxwell, D.L. y Stake E. (2006). Research and Statistical Methods in Comunication Sciences and Disorders. Michigan: Universidad de Michigan, Thomson/Delmar learning.

McCormack, B., Taylor, B., McConville, J., Slater, P. y Murray, B. (2008). The Reliability Element of the Northern Ireland Single Assessment Tool for the Health and Social Care of Older People. Northern Ireland. Belfast: Department of Health, Social Services and Public Safety.

Murillo, F. y Krichesky, G. (2012). El proceso del cambio escolar. una guía para impulsar y sostener la mejora de las escuelas. REICE. Revista Iberoamericana sobre Calidad, Eficacia y Cambio en Educación, 10(1), 26-43.

Orbegozo, A. (2016). Regulación del diagnóstico social en el País Vasco: intenciones, resultados y vericuetos. Zerbitzuan, 61, 21-32. Disponible en: http://dx.doi.org/10.5569/1134-7147.61.02

Raya, E. (2007). Exclusión social: Indicadores para su estudio y aplicación para el trabajo social. Revista del Ministerio de Ttrabajo y Asuntos Sociales, 7, 155-171.

Rodríguez, G., Camacho, J., Rodrigo, M. J., Martín, J. C. y Máiquez, M. L. (2006). Evaluación del riesgo psicosocial en familias usuarias de servicios sociales municipales. Psicothema, 18(2), 200-206. 